Paula dos Reis CorrêA ${ }^{1}$

Paulo Rodrigo dos Santos David ${ }^{2}$

Nathála Perpétua Peres ${ }^{3}$

Ketth CÁssia da CUNha ${ }^{4}$

Margarete Teresa Gottardo de Almeida ${ }^{5}$

Artigo original

Palavras-chave

Candidíase vulvovaginal/epidemiologia

Fatores de risco

Fatores de virulência

Vulvovaginite

Sinais e sintomas

Keywords

Candidiasis, vulvovaginal/epidemiologia

Risk factors

Virulence factors

Vulvovaginitis

Signs and symptoms

\title{
Caracterização fenotípica de leveduras isoladas da mucosa vaginal em mulheres adultas
}

\author{
Phenotypic characterization of yeasts isolated from \\ the vaginal mucosa of adult women
}

Resumo

OBJETIVO: caracterizar fenotipicamente leveduras isoladas do conteúdo vaginal de 223 mulheres adultas, sintomáticas (S) e assintomáticas (A) para vulvovaginite, e determinar os indicadores clínicos que possivelmente levam ao surgimento de sinais e sintomas relacionados ao acometimento da mucosa por essa patologia. MÉTODOS: inicialmente foi aplicado um questionário, com questões abertas e fechadas, sobre dados clínicos epidemiológicos. Logo, ocorreu o diagnóstico micológico com semeadura em meio Chrom Agar Candida, identificação micromorfológica e bioquímica. Métodos específicos para detecção de fatores de virulência, proteinase e fosfolipase foram empregados. A análise estatística das variáveis foi estabelecida utilizando os testes $\chi^{2}$ e $\chi^{2}$ de Pearson. RESULTADOS: Candida albicans foi a espécie mais prevalente $(87 \%$, S e $67 \%$, A), seguida de Candida glabrata $(4 \%$, S e 17\%, A). O número de mulheres que referiram adoção de anticoncepcionais foi mais alto entre as sintomáticas, 77\%. Nos dois grupos estudados, em torno de $87 \%$ apresentaram ciclos menstruais regulares, 57\% das mulheres eram casadas com idade entre 30 a 40 anos. Em relação a práticas sexuais, houve para parte das pacientes, concomitância entre os hábitos, anal, oral e vaginal. Em relação à fosfolipase, apenas Candida albicans produziu este fator de virulência em 37,5\%. A proteinase foi detectada em Candida albicans, Candida glabrata e Candida parapsilosis. Esse último fator de virulência esteve associado, principalmente, a isolados de pacientes sintomáticas. CONCLUSÕES: a colonização e infecção da mucosa vaginal por levedura é real com diversas espécies de Candida presentes. No entanto, Candida albicans se destaca como espécie prevalente em mucosa vaginal de mulheres adultas. Fica evidente a emergência de espécies de Candida não albicans, algumas com resistência intrínseca aos azólicos, tais como Candida glabrata, Candida parapsilosis, Candida tropicalis, e Candida guillermondii, o que pode ser explicado pelo uso inadequado de medicamentos e tratamento empírico.

\section{Abstract}

PURPOSE: to characterize, phenotypically, yeasts isolated from the vaginal content of 223 symptomatic (S) and asymptomatic (A) adult women with vulvovaginitis, and to determine the clinical indicators which may lead to the appearance of signs and symptoms related to the mucosa involvement by this pathology. METHODS: a questionnaire with open and closed questions on epidemiological clinical data was applied initially. Then, mycological diagnosis with sowing in Chrom Agar Candida was done, followed by micro-morphological and biochemical identification. Specific methods for the detection of the virulence factors, proteinase and phospholipase were employed. Statistical analysis was performed through $\chi^{2}$ and Pearson's $\chi^{2}$ tests. RESULTS: the most prevalent species found was Candida albicans $187 \%, \mathrm{~S}$ and $67 \%$, A) followed by Candida glabrata $(4 \%, \mathrm{~S}$ e $17 \%$ A). The number of women reporting the use of contraceptives was higher among the symptomatic, $77 \%$. In the two groups studied, about $87 \%$ of the women presented regular menstrual cycles and $57 \%$ were married with ages between 30 to 40 years old. Concerning the sexual practices, there has been concomitance among anal, oral and vaginal habits from the patients. Only Candida albicans produced the virulence factor phospholipase in $37.5 \%$ of them. Proteinase has been detected in Candida albicans, Candida glabrata and Candida parapsilosis. This latter virulence factor was mainly associated to isolates from symptomatic patients. CONCLUSIONS: it is a fact that the vaginal mucosa can be colonized and infected by yeasts, with several Candida species present. Nevertheless, Candida albicans is the most prevalent in the vaginal mucosa of adult women. It is evident the emergence of non-albicans Candida species, some of them with intrinsic resistance to azolics, such as Candida glabrata, Candida parapsilosis, Candida tropicalis, and Candida guillermondii, which can be explained by the inadequate use of medicines and empirical treatment.

Correspondência:

Paula dos Reis Corrêa Rua Padre Américo Ceppi, 680 -Brasil CEP 38400-672 - Uberlândia/MG, Brasil E-mail: falandocomapaulinha@yahoo.com.br

Serviço de Ginecologia do Hospital de Base de São José do Rio Preto - São José do Rio Preto (SP), Brasil.

'Pós-graduanda (Mestrado) em Microbiologia pela Universidade Estadual Paulista "Júlio de Mesquita Filho"- UNESP - São José do Rio Preto (SP), Brasil.

${ }^{2}$ Médico da Faculdade de Medicina de São José do Rio Preto - FAMERP - São José do Rio Preto (SP), Brasil.

${ }^{3}$ Enfermeira da Faculdade de Medicina de São José do Rio Preto - FAMERP - São José do Rio Preto (SP), Brasil.

${ }^{4}$ Bióloga da Faculdade de Medicina de São José do Rio Preto - FAMERP - São José do Rio Preto (SP), Brasil.

${ }_{5}^{5}$ Professora Doutora de Microbiologia da Faculdade de Medicina de São José do Rio Preto - FAMERP - São José do Rio Preto (SP), Brasil. 


\section{Introdução}

A candidíase vulvovaginal (CVV) é uma infecção da mucosa genital ${ }^{1}$, causada por fungos leveduriformes ${ }^{2}$. Esses micro-organismos são considerados oportunistas porque em condições propícias, como presença de fatores pré-disponentes locais ou sistêmicos, podem proliferar e desencadear processos infecciosos ${ }^{3}$.

A flora vaginal autóctone é complexa e sua composição varia de acordo com uma multiplicidade de acontecimentos, como fatores hormonais; número de parceiros; uso de contraceptivos orais; uso de antibioticoterapia; diabetes; uso de preservativos; tabagismo e maus hábitos em matéria de higiene. Todos estes são considerados como fatores de risco para o surgimento de infecções genitais ${ }^{4}$.

As cepas do gênero Candida podem ser encontradas como habitantes normais dessas mucosas: estando presente em 20 a $80 \%$ da população adulta saudável ${ }^{5}$. Entretanto, são detectados em $10 \%$ das mulheres na pré-menopausa, 5 a $10 \%$ das menopausadas e em $30 \%$ das gestantes ${ }^{6}$. Durante o período entre a menarca e a menopausa, aproximadamente $75 \%$ das mulheres sofrem ao menos um episódio de CVV. Dessas, $40 \%$ tiveram mais de um episódio e $5 \%$ apresentam episódios de repetição com quatro ou mais eventos anuais, daí a designação de CVV recorrente ${ }^{7}$. As manifestações clínicas mais comuns nesse tipo de infecção são disúria, hiperemia, ardência, corrimento, prurido, dispareunia e fissuras ${ }^{8}$.

Estudos sobre a prevalência de CVV com diagnóstico pós-cultura indicam que, embora a espécie mais frequente seja Candida albicans, com $80 \%$ de ocorrência, a frequência de outras espécies "não albicans", está aumentando", e nesse grupo, foram encontradas principalmente Candida tropicalis, Candida glabrata e Candida krusei ${ }^{10}$. Tal fato é preocupante, pois em geral as duas últimas são mais resistentes aos antifúngicos clássicos utilizados na prática clínica ${ }^{1}$.

Apesar da alta prevalência de mulheres adultas com queixa clínica sugestiva de CVV, até o momento, não existem estudos sistemáticos sobre sua etiologia na região do Noroeste paulista. Estudos que abordem a origem, bem como fatores de riscos associados à vulvovaginite, contribuirão para adoção de estratégias de medida de controle dessa nosologia. Assim, a presente proposta teve como objetivo geral investigar os agentes fúngicos presentes na mucosa vaginal de mulheres adultas de São José do Rio Preto, região Sudeste do Brasil.

\section{Métodos}

Foi realizado um estudo transversal de caráter exploratório, com abordagem quantitativa, durante o período de Março de 2007 a Agosto de 2008. Após aprovação pelo Comitê de Ética em Pesquisa da Faculdade de Medicina de São José do Rio Preto, e assinatura do termo de consentimento pelas pacientes, o trabalho teve seu início. Inicialmente, os dados foram obtidos por meio de entrevista, a qual incluiu questões abertas e fechadas sobre dados clínicos epidemiológicos e outras pertinentes a variáveis envolvidas: idade, escolaridade, práticas sexuais, método anticoncepcional adotado e uso prévio de antibióticos, além da análise de fatores predisponentes para CVV, seguido da investigação micológica. Para tal, participaram do estudo 223 mulheres adultas, sendo caracterizadas como assintomáticas (101) e sintomáticas (122) para CVV. A amostragem não foi populacional e, sim, proveniente de ambulatório de ginecologia, isto é, por demanda espontânea.

A coleta das amostras e a avaliação clínica foram estabelecidas por médicos do Serviço de Ginecologia do Hospital de Base de São José do Rio Preto. Parte do conteúdo do fluido vaginal das 223 mulheres foi utilizado para o exame direto e outra, para cultura em Ágar Sabouraud Dextrose (Himedia, Mumbai, India) com e sem 100 mg/ $\mathrm{mL}$ de cloranfenicol (Difco, Lawrence, EUA). Seguiu-se a incubação a $30{ }^{\circ} \mathrm{C}$ por até 15 dias, sendo que as culturas que não apresentaram crescimento, após esse período, foram consideradas negativas.

Inicialmente, a identificação ocorreu segundo características morfológicas e bioquímicas em Chrom Ágar Candida ${ }^{\circledR}$ (Paris, França), este teste fornece dados presuntivos de identificação, a partir da diferenciação de coloração e morfologia das colônias por espécies. A confirmação das espécies foi estabelecida por auxanogra$\mathrm{ma}^{11}$, uma prova de assimilação de açúcares, realizada em meio mínimo de carbono e nitrogênio. No momento da leitura segue-se a uma chave de identificação, observando na placa os açúcares assimilados até chegar à espécie. O microcultivo em ágar fubá (Oxoid, Basingstok, UK) acrescido de twen 80 (Vetec, Rio de Janeiro, Brasil) e a prova do tubo germinativo, foram realizados como testes conclusivos para identificação de Candida albicans, já que essa é a única espécie que apresenta resultado positivo nos dois testes.

Todas as leveduras foram avaliadas quanto à produção de fatores de virulência, a saber, fosfolipase e proteinase, segundo protocolos descritos ${ }^{12,13}$. Estes testes foram realizados em duplicata e armazenados em estufa úmida a $35^{\circ} \mathrm{C}$, por até cinco dias, as leituras foram realizadas observando a presença de halos ao redor das colônias, indicativos para produção de tipos enzimáticos.

Todas as variáveis foram analisadas pelo teste de associação do tipo $\chi^{2}$ com intervalo de confiança de $95 \%$. Para associação entre produção de fatores de virulência e dado clínico (sintomática ou assintomática), aplicou-se o teste do $\chi^{2}$ de Pearson. 


\section{Resultados}

\section{Caracterização micológica}

Das 223 participantes do estudo, 87 mulheres apresentaram cultura positiva para leveduras, sendo $69(55 \%)$ isolados provenientes do Grupo Sintomático $(\mathrm{n}=122)$, e $18(18 \%)$ do Assintomático $(\mathrm{n}=101)$. A distribuição das espécies fúngicas e o correspondente percentual de ocorrência estão apresentados na Figura 1.

A análise individual dos grupos possibilitou a detecção de diferenças quanto aos critérios clínicos e epidemiológicos. Assim, no Grupo Sintomático a média de idade foi de 30,6 anos; $57 \%$ eram casadas, $36 \%$ separadas e $7 \%$ solteiras. Quanto ao método anticoncepcional, foi observado que $77 \%$ referiram adoção, sendo $46,5 \%$ usuárias de anticoncepcional hormonal oral, isolado ou combinado com outro método. A recorrência foi observada em $84,2 \%$ dos casos, sendo que desses $69 \%$ tiveram até cinco episódios e $3 \%$ mais do que cinco. Considerando o ciclo menstrual, $88 \%$ menstruavam de forma regular. Em relação à atividade sexual, a prática vaginal e a oral foram as mais frequentes. As demais modalidades, oral, anal e ou concomitância entre duas ou mais, ocorreram em menor percentual. Diversos sinais e sintomas concorreram para manifestação clínica de CVV, com destaque para a disúria $(91,3 \%)$ e hiperemia vaginal $(89,6 \%)$ como as mais frequentes.

Para o Grupo Assintomático, a média de idade foi de 37,1 anos; $56 \%$ eram casadas, $32 \%$ solteiras e
$12 \%$ separadas. A adoção de métodos anticoncepcionais esteve presente em $44 \%$ das participantes, sendo o tipo hormonal oral isolado ou associado com outros métodos observado em $28,7 \%$. Os resultados relativos a episódios anteriores de CVV foram detectados em $42 \%$ das pacientes, com $71 \%$ para até cinco episódios e $29 \%$ para mais do que essa quantidade. Quanto ao ciclo menstrual, $72 \%$ menstruavam, sendo $87 \%$ de modo regular. A avaliação da atividade sexual mostrou que as práticas vaginal e oral foram preferenciais. Entretanto, outras práticas, oral, anal e ou concomitância entre duas ou mais, ocorreram em menor percentual. A detecção de espécies não albicans foi mais frequente no Grupo Assintomático, totalizando $33 \%$.

Frente aos isolados clínicos de Candida sp., estudou-se a produção de fator de virulência, proteinase e fosfolipase, comumente associados à patogenicidade. Assim, C. albicans foi a única espécie produtora da fosfolipase. Entretanto para a mesma espécie, a produção concomitante deste fator e a proteinase foram observadas em 33,3\% ( $n=20)$. Nos isolados de Candida albicans, dentro do Grupo Sintomático, os resultados de produção de fator de virulência foram mais expressivos: proteinase ocorreu em $66,7 \%(n=40)$ e fosfolipase em 37,5\% ( $\mathrm{n}=23$ ). Já para Candida glabrata, a síntese de proteinase ocorreu apenas nos isolados provenientes do Grupo Assintomático, correspondendo a $16,7 \%(\mathrm{n}=1)$.

As cepas de Candida tropicalis demonstraram ocorrência de $100 \%(\mathrm{n}=2)$ de produção do fator proteinase. Já a mesma enzima nos isolados de Candida parapsilosis ocorreu

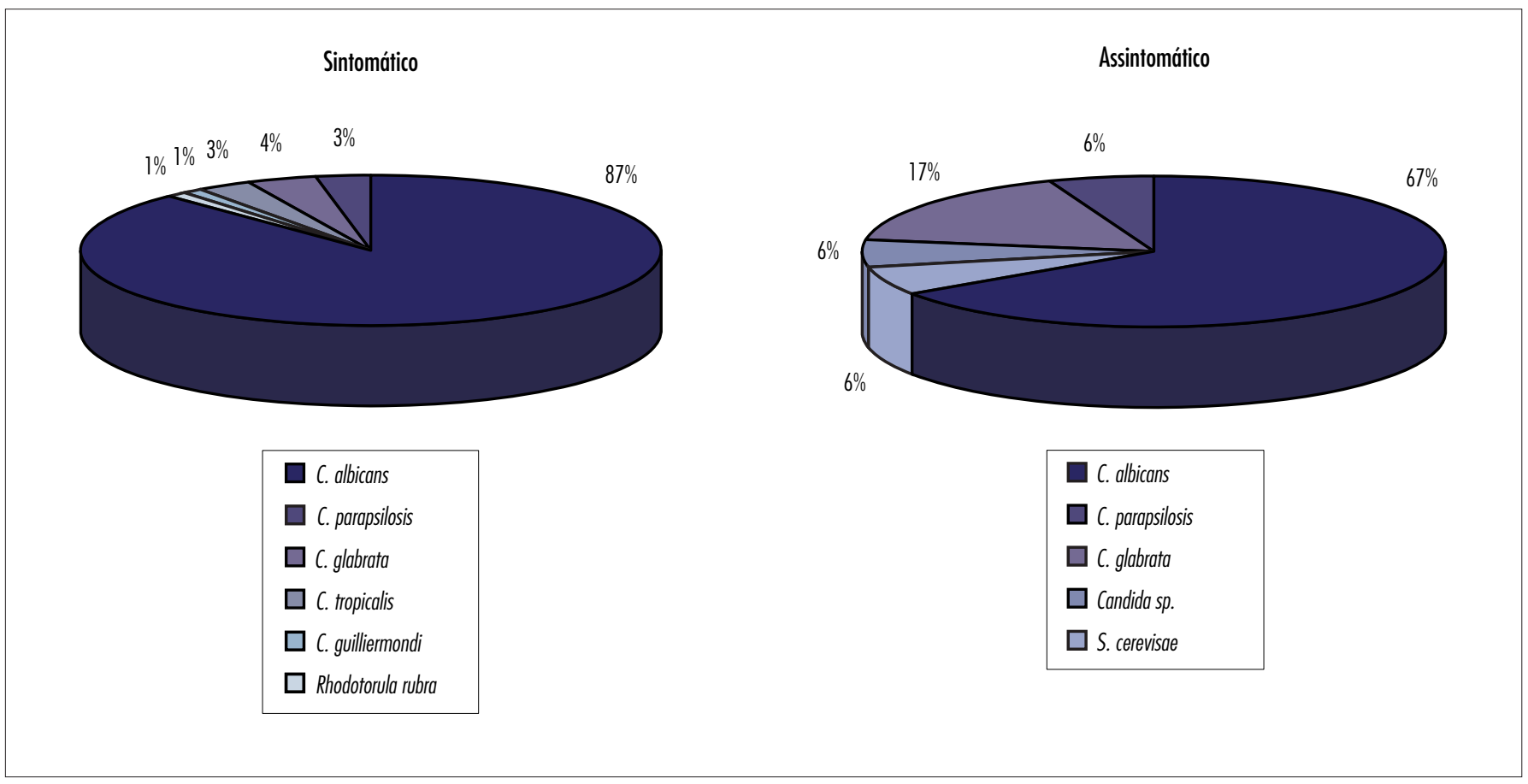

Figura 1 - Distribuição da frequência das espécies de leveduras no conteúdo vaginal das mulheres adultas, sintomáticas e assintomáticas. 
em três casos, sendo originários do Grupo Sintomático $(\mathrm{n}=1)$ e Grupo Assintomático $(\mathrm{n}=2)$.

Para as espécies Rhodotorula rubra, Saccharomyces cerevisae, Candida spp. e Candida guilliermondii, independentemente do grupo, não foi detectado nenhum dos fatores.

De acordo com o teste $\chi^{2}$ de Pearson, não houve evidência de associação entre a condição clínica, sintomática ou não, com produção de fosfolipase $(\mathrm{p}=0,1)$. Porém, houve frente à proteinase $(\mathrm{p}=0,02)$, uma vez que as cepas isoladas das mulheres sintomáticas foram produtoras desse fator de virulência em 59,8\% dos casos.

\section{Discussão}

Infecções por Candida sp em superfícies mucosas são comuns, debilitantes e frequentemente recorrentes. Aproximadamente três quartos de mulheres saudáveis em idade fértil sofrem de CVV com significante morbidade física e psicológica ${ }^{14}$. Na presente análise, foi possível identificar Candida sp em 31\% de mulheres sintomáticas e em $8 \%$ de mulheres assintomáticas.

Cerca de $80 \%$ de todas as leveduras identificadas na mucosa vaginal são Candida albicans ${ }^{15}$. De fato, Candida albicans esteve amplamente distribuída na população estudada, ora como comensal $(67 \%)$ ou como patógena (87\%). Uma provável justificativa para o alto índice de Candida albicans, presente na mucosa de mulheres assintomáticas, seria a presença desta espécie no trato gastrintestinal ou na via sexual, na qual a partir dessa fonte endógena, disseminaria ${ }^{16}$.

Entretanto, recentemente, as espécies de Candida não albicans vêm mudando este panorama clínico, apresentando-se como patógeno, especialmente pela seleção de espécies mais resistentes ${ }^{17}$. A porcentagem de ocorrência por espécies não albicans foi expressiva, $13 \%$ ao Grupo Sintomático e $33 \%$ ao Assintomático. Outros estudos confirmam esta distribuição, foi observada a ocorrência destas espécies entre 9,9 a 32\% dos casos de vulvovaginite, respectivamente. As espécies de não albicans encontradas aqui foram Candida glabrata, Candida tropicalis, Candida guillermondi e Candida parapsilosis, dado concordante com outros estudos, que as identificam na última década, como espécies emergentes responsáveis por $\mathrm{CVV}^{1,18}$. Chamase a atenção que espécies não albicans vêm ocorrendo em mulheres adultas sem sintomatologia, descrito pela literatura em cerca de $44 \%$ dos $\operatorname{casos}^{19}$. Tendo em vista a presente investigação, os dados foram concordantes com o exposto acima.

O aumento da acidez vaginal é influenciado por diversos fatores, dentre estes, a adoção de anticoncepcionais hormonais orais, especialmente em estado hiperestrogênico. $\mathrm{O}$ uso de anticoncepcionais oral fornece condições nutritivas que favorecem o estabelecimento do fungo na mucosa vaginal ${ }^{20}$.
No presente estudo, a maior parte das mulheres portadoras de leveduras fazia uso desse método contraceptivo.

As formas recorrentes são definidas com a repetição de quatro ou mais episódios sintomáticos ao ano, ou quando se tem três episódios não relacionados com antibioticoterapia prévia no último ano ${ }^{7}$. Nesse sentido, das mulheres acometidas com CVV, 10 a $40 \%$ tornam-se recorrentes ${ }^{21}$. No presente estudo, foram detectados índices maiores aliados aos dos Grupos Assintomático (42\%) e Sintomático $(84,2 \%)$. O significado de Candida sp., presente na vagina de mulheres assintomáticas entre episódios de recorrência, não está claro. $\mathrm{O}$ achado desses organismos, durante períodos livres de sintomas, poderia indicar um tratamento prévio inadequado ao uso insuficiente de antifúngicos, ou mesmo à resistência dos fungos aos fármacos. Entre $10 \mathrm{a}$ $33 \%$ dos casos de recorrência descritos na literatura ${ }^{22,23}$, a etiologia é atribuída a espécies não albicans, como Candida glabrata (mais frequente), seguida de Candida krusei, Candida parapsilosis e Saccharomyces cerevisae, dados equivalentes aos encontrados nesse estudo.

O ciclo menstrual é qualificado como regular quando da sua ocorrência entre 25 e 35 dias. Essa regularidade, considerando o intervalo de análise dos últimos seis meses, foi detectada em $88 \%$ das mulheres sintomáticas e $87 \%$ das assintomáticas. Embora não haja consenso no que tange aos fatores de risco para a CVV, a presença de ciclos menstruais regulares tem sido identificada como relevante, uma vez que a acidez, subsequente aos picos hormonais de FSH, LH, estradiol e progesterona determinantes da ovulação e formação do corpo lúteo, poderia contribuir para invasão tecidual do fungo ${ }^{22}$.

Diversos sinais e sintomas estão presentes na maioria das mulheres com CVV, com destaque para prurido, dispareunia e corrimento, com índices entre 82 a 94\%, e modernamente associados à espécie Candida albicans ${ }^{21}$. Comparando os resultados aqui obtidos com os da literatura, as mesmas queixas clínicas foram observadas nas participantes do Grupo Sintomático, porém com índices menores: $41,7,17,4$ e 55,6\%, respectivamente. Entretanto, disúria e hiperemia vaginal ocorreram em altos índices. Ainda, comparando as queixas entre os dois grupos analisados (Sintomático e Assintomático), foi observada uma diferença significante apenas para disúria, hiperemia, dispareunia, hiperemia vulvar e fissura. De fato, corrimento e prurido podem estar associados a outros fatores, desde microbianos, imunológicos e fisiológicos ${ }^{16}$.

A concomitância entre práticas sexuais tem sido considerada como fator de risco para o estabelecimento da infecção fúngica, o que foi confirmado com os dados dessa investigação. Os resultados mostraram associação significante entre sexo orogenital e infecção por Candida, dados suportados por estudo prévio ${ }^{23}$. Uma explicação 
para tal fato deve ser a transmissão por contato, além de que um terço da população adulta pode conter Candida albicans oral e, consequentemente, outras espécies ${ }^{24}$.

Os atributos que contribuem para virulência de Candida albicans incluem adesão, formação de hifas e produção de enzimas hidrolíticas extracelulares ${ }^{25,26}$. De fato, em relação às enzimas, Candida albicans produz fosfolipase $\mathrm{e}$ proteinase, e a detecção desses tipos enzimáticos comprova a alta patogenicidade dessa espécie, especialmente nas de origem do Grupo Sintomático.
Concluímos que a colonização e infecção da mucosa vaginal por levedura é real com diversas espécies de Candida presentes. No entanto, Candida albicans se destaca como espécie prevalente em mucosa vaginal de mulheres adultas. Fica evidente a emergência de espécies de Candida não albicans, algumas com resistência intrínseca aos azólicos, tais como Candida glabrata, Candida parapsilosis, Candida tropicalis e Candida guillermondii, o que pode ser explicado pelo uso inadequado de medicamentos e tratamento empírico.

\section{Referências}

1. Ferrazza MHSH, Maluf MLF, Consolaro MEL, Shinobu CS, Svidzinski TIE, Batista MR. Caracterização de leveduras isoladas da vagina e sua associação com candidíase vulvovaginal em duas cidades do sul do Brasil. Rev Bras Ginecol Obstet. $2005 ; 27(2): 58-3$

2. Geva A, Bornstein J, Dan M, Shoham HK, Sobel JD. The VI-SENSEvaginal discharge self-test to facilitate management of vaginal symptoms. Am J Obstet Gynecol. 2006;195(5):1351-6.

3. Silva CRG, Melo KE, Leão MVP, Ruis R, Jorge AOC. Presença de Candida nas mucosas vaginal e bucal e sua relação com $\lg A$ salivar. Rev Bras Ginecol Obstet. 2008;30(6):300-5.

4. Campos AC, Freitas-Junior R, Ribeiro LF, Paulinelli RR, Reis C. Prevalence of vulvovaginitis and bacterial vaginosis in patients with koilocytosis. Sao Paulo Med J. 2008;126(6):333-6.

5. Rosa MI, Rumel D. Fatores associados à candidíase vulvovaginal: estudo exploratório. Rev Bras Ginecol Obstet. 2004;26(1):65-7.

6. Schaller M. [Candida albicans - interactions with the mucosa and the immune system]. J Dtsch Dermatol Ges. 2006;4(4):328-36.

7. Fidel PL Jr. History and update on host defense against vaginal candidiasis. Am J Reprod Immunol. 2006;57(1):2-12.

8. Edwards $L$. The diagnosis and treatment of infectious vaginitis. Dermatol Ther. 2004;17(1):102-10.

9. García Heredia M, García SD, Copolillo EF, Cora Eliseth M, Barata $A D$, Vay $C A$, et al. Prevalence of vaginal candidiasis in pregnant women. Identification of yeasts and susceptibility antifungal agents. Rev Argent Microbiol. 2006;38(1):9-12.

10. Zardo V, Mezzari A. Os antifúngicos nas infecções por Candida sp. News Lab. 2004;63:136-6.

11. Larone DH. Medically important fungi. A guide to identification. AMS Press: Washington; 1995.

12. Price MF, Wilkison ID, Gentry IO. Plate method for detection of phospholipase activity in Candida albicans. Saboraudia. 1982;20:15-20.

13. Ruchel R, De Bernardis F, Ray TL, Sullivan PA, Coleg. GT. Candida acid proteinases. J Med Vet Mycol. 1992; 30 (Suppl 1):123-132.

14. Boatto HF, Moraes MS, Machado AP, Girão MJBC, Fischman O. Correlação entre os resultados laboratoriais e os sinais e sintomas clínicos das pacientes com candidíase vulvovaginal e relevância dos parceiros sexuais na manutenção da infecção em São Paulo, Brasil. Rev Bras Ginecol Obstet. 2007;29(2):80-4.
15. Benavides JL, Rodriguez DS, Menchaca RT, Gonzalez G, Gonzalez EG, Tristan ER. Especies de Candida no albicans en la consulta de ginecología. Med Universitaria. 2007;9(37):161-5.

16. Mendling W, Pinto De Andrade M, Gutschmidt J, Gantenberg R, Presber W, Schönian $G$. Strain specificity of yeasts isolated from different locations of women suffering from vaginal candidosis, and their partners. Mycoses. 2000;43(1 1-12):387-92.

17. Nyirjesy P, Seeney SM, Grody MH, Jordan CA, Buckley HR. Chronic fungal vaginitis: the value of cultures. Am J Obstet Gynecol. 1995; 173(3 Pt 1):820-3.

18. Rylander E, Berglund AL, Krassny C, Petrini B. Vulvovaginal candida in a young sexually active population: prevalence and association with oro-genital sex and frequent pain at intercourse. Sex Transm Infect. 2004;80(1):54-7.

19. Holanda AAR, Fernandes ACS, Bezerra CM, Ferreira MAF, Holanda MRR, Holanda JCP, et al. Candidíase vulvovaginal: sintomatologia, fatores de risco e colonização anal concomitante. Rev Bras Ginecol Obstet. 2007;29(1):3-9.

20. Dan M, Kaneti N, Levin D, Poch F, Samra Z. Vaginitis in a gynecologic practice in Israel: causes and risk factors. Isr Med Assoc J. 2003;5(9):629-32.

21. Ferrer J. Vaginal candidosis: epidemiological and etiological factors. Int J Gynaecol Obstet. 2000;71 Suppl 1:S21-7.

22. Spinillo A, Bernuzzi AM, Cevini C, Gulminetti R, Luzi S, De Santolo $A$. The relationship of bacterial vaginosis, Candida and Trichomonas infection to symptomatic vaginitis in postmenopausal womem attending a vaginitis clinic. Maturitas. 1997;27(3):253-60.

23. Patel DA, Gillespie J, Sobel JD, Leaman D, Nyirjesy M, Weitz MV, et al. Risk factors for recurrent vulvovaginal candidiasis in women receiving maintenance antifungal therapy: results of a prospective cohort study. Am J Obstet Gynecol. 2005; 190(3):644-53.

24. Reed BD, Gorenflo DW, Gillespie BW, Pierson CL, Zazove P. Sexual behaviors and other risk factors for Candida vulvovaginitis. J Womens Health Gend Based Med. 2000;9(6):645-55.

25. Monod M, Borg-von ZM. Secreted proteinases and other virulence mechanisms of Candida albicans. Chem Immunol. 2002;81:11428

26. Ombrella AM, Racca L, Ramos L. Actividades proteinasa y fosfolipasa de aislamientos de Candida albicans provenientes de secreciones vaginales con distintos valores de $\mathrm{pH}$. Revista Iberoam Mícol. 2008;25(1):12-16. 